Check for updates

Cite this: RSC Adv., 2018, 8, 39013

Received 20th August 2018

Accepted 14th November 2018

DOI: $10.1039 / c 8 r a 06943 c$

rsc.li/rsc-advances

\section{Fabrication of a novel whole tissue-engineered intervertebral disc for intervertebral disc regeneration in the porcine lumbar spine $\uparrow$}

\author{
Fei Yang, (D) ab Dongqin Xiao, (D) *a Qiao Zhao, ${ }^{\mathrm{c}}$ Zhu Chen, ${ }^{a}$ Kang Liu, ${ }^{\text {a }}$ Shixiao Chen, ${ }^{d}$ \\ Xiao Sun, ${ }^{a}$ Qiuju Yue, ${ }^{a}$ Ruolan Zhang ${ }^{a}$ and Gang Feng*ac
}

\begin{abstract}
Tissue-engineered intervertebral discs (IVDs) have been proposed as a useful therapeutic strategy for the treatment of intervertebral disc degeneration (IDD). However, most studies have focused on fabrication and assessment of tissue-engineered IVDs in small animal models and the mechanical properties of the scaffolds are far below those of native human IVDs. The aim of this study was to produce a novel tissueengineered IVD for IDD regeneration in the porcine lumbar spine. Firstly, a novel whole tissueengineered IVD scaffold was fabricated using chitosan hydrogel to simulate the central nucleus pulposus (NP) structure, surrounded with a poly(butylene succinate-co-terephthalate) (PBST) fiber film for inner annulus fibrosus (IAF). And, a poly(ether ether ketone) (PEEK) ring was used to stimulate the outer annulus fibrosus (OAF). Then, the scaffolds were seeded with IVD cells and the cell-scaffold hybrids were transplanted into the porcine damaged spine and harvested at 4 and 8 weeks. In vitro cell experiments showed that IVD cells distributed and grew well in the scaffolds including porous hydrogel and PBST fibers. After implantation into pigs, radiographic and MRI images indicated that the tissue-engineered IVD construct could preserve the disc height in the case of discectomy as the normal disc height and maintain a large extracellular matrix and water content in the NP. Combined with the histological and gene expression results, it was concluded that the tissue-engineered IVD had similar morphological and histological structure to the natural IVD. Moreover, after implantation for 8 weeks, the tissue-engineered IVD showed a good compressive stress and elastic moduli, approaching those of natural porcine IVD. Therefore, the prepared tissue-engineered IVD construct had similar morphological and biofunctional properties to the native tissue. Also, the tissue-engineered IVD construct with excellent biocompatibility and mechanical properties provides a promising candidate for human IDD regeneration.
\end{abstract}

\section{Introduction}

Intervertebral disc degeneration (IDD) is regarded as the leading factor in lower back pain (LBP), which imposes an enormous clinical and economic burden on society. ${ }^{1}$ In the United States, the costs associated with LBP range from $\$ 19$ to $\$ 118$ billion per year and continue to increase as the population ages. $^{2}$ Currently, the main medical interventions available are conservative treatment and surgical management. However, the conservative treatments (such as pain-relieving

${ }^{a}$ Research Institute of Tissue Engineering and Stem Cells, Nanchong Central Hospital, The Second Clinical College of North Sichuan Medical College, Nanchong, Sichuan, 637000 China. E-mail: xdqdxs@163.com; fenggangncch@163.com

${ }^{b}$ Orthopedics, Nanchong Central Hospital, Nanchong, Sichuan, 637000 China ${ }^{c}$ Orthopedics, Southwest Medical University, Luzhou, Sichuan, 646000 China ${ }^{d}$ Radiology Department, Nanchong Central Hospital, Nanchong, Sichuan, 637000 China

† Electronic supplementary information (ESI) available. See DOI: 10.1039/c8ra06943c pharmaceuticals, physical manipulation and electric stimulus) work only at the early stage of the IDD, aiming at alleviating clinical symptoms. ${ }^{3}$ When these nonoperative approaches fail, surgical managements (such as lumbar fusion, discectomy and total disc arthroplasty) are performed to directly remove the source of pain and restore the biomechanical function of discs in the short term. Nevertheless, these interventions do not address the biological basis, and further induce degeneration in the diseased or adjacent motion segments or failure due to the biomechanical changes between the implant and the remaining spine tissues in the long term. ${ }^{4}$ Therefore, developing effective therapies for IDD treatment is urgently needed.

For addressing the biological basis for the disease and preventing degeneration, full understanding of intervertebral disc (IVD) structure appears to be particularly important. The IVD is a fibrocartilaginous tissue composed of three distinct regions, including nucleus pulposus (NP), annulus fibrosus (AF) and cartilage endplates (Fig. 1). The collagenous AF surrounds a central, highly hydrated NP, and these structures are separated from the adjacent vertebral body by the inferior and 


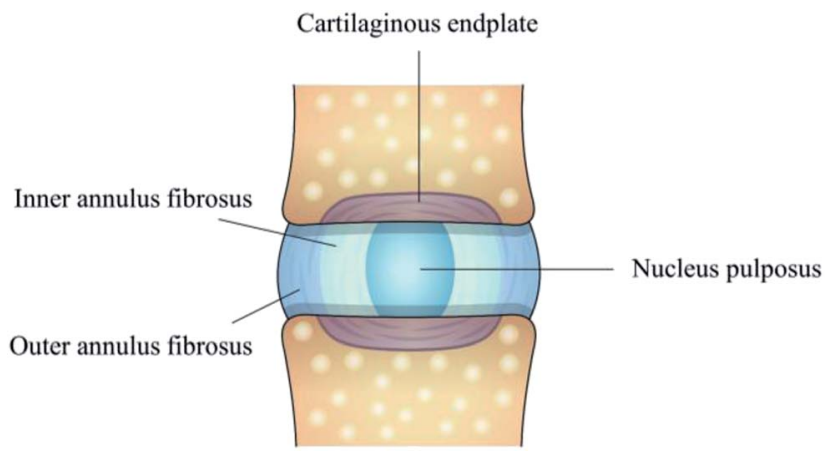

Fig. 1 Schematic illustration of IVD structure composed of nucleus pulposus, inner annulus fibrosus, outer annulus fibrosus and cartilage endplates.

superior cartilage endplates. AF, being further divided into the inner annulus fibrosus (IAF) and outer annulus fibrosus (OAF), resists tensile forces generated by twisting and bending and keeps the NP in its position, while NP possesses large amounts of water to provide resistance to compression. IVD aging and overloading inevitably cause NP degeneration, a decrease in cellularity and alternations in extracellular matrix (ECM) composition, which ultimately affect the morphology and biomechanical function of the whole IVD. Therefore, treatment strategies, such as stem cell and gene therapies, ${ }^{5,6}$ have been developed for alleviating the pain and degenerative process in the early stage. However, for the late stage of IDD, a more comprehensive approach is required to preserve the integrity of whole disc structure and mechanical function.

Recently, tissue engineering aiming to repair or replace damaged tissues has gained great attention in restoring the biological function of IDD. ${ }^{7,8}$ Researchers have attempted to construct tissue engineered $\mathrm{NP}^{9,10}$ or $\mathrm{AF}^{11,12}$ for displacing the impaired NP or AF alone. Despite substantial progress in developing tissue-engineered NP and AF, none of them has translated into clinical implementation. This could be due to lack of effective strategies to fabricate the whole tissueengineered IVD, since the NP or AF regeneration strategies alone cannot effectively prevent IDD in severe case. ${ }^{13}$ Considering that IDD usually involves both central NP and surrounding AF, which need to be repaired simultaneously to maintain the biofunction of IVD, so fabrication of the whole tissue-engineered IVD via a combination of tissue-engineered $\mathrm{NP}$ and $\mathrm{AF}$ is indispensable. To this end, the researches about tissue-engineered IVD composed of NP and AF components, such as simultaneously using bone matrix gelatin (BMG) for the AF phase and acellular cartilage ECM for the inner NP phase or electrospun poly(E-caprolactone) (PCL) scaffold for AF replacement and hydrogels for NP replacement, ${ }^{14,15}$ have been reported recently. However, the two primary limitations are present in these studies. Firstly, the mechanical stiffness of materials used for AF scaffolds, such as BMG and PCL, is far below that of native AF. The use of these materials only can contain a fluid NP but may not bear twisting and bending loads. Secondly, most of these studies focus on small animals with lower loads, which dramatically differ from human physiological loading environment. Thus, developing tissue-engineered IVD has focused on mimicking the biochemical and biomechanical properties of human native IVD in these days.

Therefore, for the constructs to be success in the translation to clinical practice, the tissue-engineered IVD need to be tailored to the degenerative state of the disc in the preclinical animal models. ${ }^{16}$ Compared with small animals (e.g. mouse, rat and rabbit), large animal models (e.g. sheep, canine and pig) have many similarities in morphology to the human IVD as well as sufficient disc height to simulate the challenging nutritional and loading environment present in human discs. ${ }^{16,17}$ Thus, the aim of this study was to produce a novel whole tissueengineered IVD consisted of triphasic scaffold for IDD regeneration in the porcine lumbar spine.

\section{Experimental}

\section{Preparation of IVD scaffold via a combination of NP and AF phase}

Firstly, chitosan hydrogel scaffold as NP phase was prepared according to our previous publication. ${ }^{18}$ Briefly, the crosslinker synthesized by the reaction between 1,6-diisocyanatohexan and polyethylene glycol-400, was added into $1 \mathrm{wt} \%$ ethylene glycol chitosan solution at the volume of $1: 100$. After several minutes at room temperature, the chitosan hydrogel was obtained. Then, the hydrogel was cut into cylindrical shape $(4 \times 4 \times 4$ $\mathrm{mm}$ ), lyophilized and stored at $4{ }^{\circ} \mathrm{C}$.

Secondly, the scaffolds for $\mathrm{AF}$ phase were prepared by introducing poly(butylene succinate-co-terephthalate) (PBST, Nanjing Yongshao Co. Ltd., China) as the IAF phase and pol$y$ (ether ether ketone) (PEEK, Victrex Co. Ltd., United Kingdom) as the OAF phase. Briefly, PBST was dissolved into chloroform to form a $25 \mathrm{wt} \%$ solution, followed by extrusion at a rate of $2 \mathrm{~mL} \mathrm{~h}^{-1}$ under $10 \mathrm{kV}$ voltages. The produced fibers were drawn across a $20 \mathrm{~cm}$ air gap onto a collecting drum. Then, the PBST fibers were collected, dried under vacuum at room temperature and stored at $4{ }^{\circ} \mathrm{C}$. Finally, the PBST film as IAF phase was then cut into long strips for further use. For PEEK scaffold, PEEK powder was placed in designed tubular molds (internal diameter: $1.4 \mathrm{~cm}$, external diameter: $1.8 \mathrm{~cm}$, height: $0.5 \mathrm{~cm}$ ). Then, the molds were heated at $150{ }^{\circ} \mathrm{C}$ under a loading pressure of $35 \mathrm{MPa}$ for $10 \mathrm{~min}$, and further heated at $375^{\circ} \mathrm{C}$ under a loading pressure of $15 \mathrm{MPa}$ for $10 \mathrm{~min}$. Afterwards, the PEEK samples were air cooled to $150{ }^{\circ} \mathrm{C}$ and removed from the molds. The obtained PEEK ring was used as the OAF phase.

Finally, to mimic the natural structure of IVD, PBST strips (as the IAF) were rolled over and inserted into PEEK ring (as the $\mathrm{OAF}$ ) to form the AF phase. Then, the NP phase composed of chitosan hydrogel was embedded into the AF phase to construct the tissue-engineered IVD scaffold.

\section{Characterization of IVD scaffolds}

The morphologies of the chitosan hydrogel, PBST film and PEEK rings were observed using scanning electron microscopy (SEM, JEOL JSM7001F). The chemical compositions of PBST 
and PEEK were analyzed using Fourier transform infrared spectroscopy (FTIR, Nicolet 5700) and X-ray Photoelectron Spectrometer (XPS, XSAM800, Kratos, UK). The crystal structure of PBST and PEEK were analyzed using X-ray diffraction (XRD, X'PertPro MPD, CuK $\alpha, 35 \mathrm{~mA}, 45 \mathrm{kV}$ ). The porosity of PBST film was calculated according to the following equation:

$$
\text { Porosity }=\left(1-\frac{\rho}{\rho_{0}}\right) \times 100 \%
$$

where $\rho$ represents the density of the electrospun PBST film and $\rho_{0}$ represents the density of bulk PBST. The density of PBST film was determined by measuring the weight by the volume of the samples. For measuring the volume of PBST film, they were cut into rectangular shapes (length: $3 \mathrm{~cm}$, width: $1 \mathrm{~cm}$, thickness: $0.15 \mathrm{~mm}$ ) and weighted. Each measurement was repeated five times. The water absorption of PBST film was calculated according to the following equation:

$$
\text { Water absorption }=\frac{W_{\mathrm{t}}-W_{\mathrm{o}}}{W_{\mathrm{o}}} \times 100 \%
$$

where $W_{\mathrm{t}}$ represents the weight of wet film and $W_{\mathrm{o}}$ represents the weight of initial dry film. For measuring the wet weight of PBST film, the sample was immersed into the phosphate buffered saline (PBS, 0.01 M) solution and removed until a constant weight was reached. Afterwards, the wet sample was placed on a paper towel to drain off the excess water and then weighed. Each measurement was repeated five times.

The stress-strain analysis of PBST film was measured using a universal test machine (Instron 5567, Instron Co., Massachusetts) as previously reported by Shao et al. ${ }^{19}$ Briefly, the samples were trimmed into dumbbell shape (type 5-D638) with the dimensions of $7.5 \mathrm{~cm} \times 0.4 \mathrm{~cm}$ and the gauge length of $2.5 \mathrm{~cm}$. The distance between the gripping points was $4 \mathrm{~cm}$. The tensile testing was carried out with a speed of $5 \mathrm{~mm} \mathrm{~min}^{-1}$. The average data of tensile strength at the breaking point was calculated for PBST film after five measurements. In addition, the tensile strength of PEEK was measured as above.

\section{Isolation and culture of porcine IVD cells}

The IVD cells were isolated as previously described by Melrose et $a .^{20}$ Briefly, freshly excised IVD tissue from porcine lumbar IVD were digested using a mixture of $0.2 \mathrm{w} / \mathrm{v} \%$ pronase (Streptomyces griseus, Calbiochem-Novabiochem Corp., USA) and $0.01 \mathrm{w} / \mathrm{v} \%$ deoxyribonuclease I (DNAase-I, Sigma) for $90 \mathrm{~min}$, followed by incubation overnight in $0.05 \mathrm{w} / \mathrm{v} \%$ collagenase II (Sigma) and 0.01 w/v\% DNAase-I in Dulbecco's Modified Eagle's Medium (DMEM-F12, Gibco, New York, USA) supplemented in $10 \%$ fetal calf serum (FCS, Gibco, New York, USA) at $37^{\circ} \mathrm{C}$. Then, the cell suspensions were filtered (70 $\mu \mathrm{m}$ cell strainer) and washed with DMEM-F12 medium. The obtained cells were expanded up to passage 2 in monolayer using DMEM-F12 medium containing $10 \% \mathrm{FCS}$ at $37{ }^{\circ} \mathrm{C}$ and $5 \% \mathrm{CO}_{2}$.

For evaluation of the obtained cells, the cells morphologies were observed using inverted phase contrast microscope (Leica model: DM IRB). For Safranin-O staining, cells were fixed with $4 \%$ paraformaldehyde for $30 \mathrm{~min}$, stained with fast green for
$1 \mathrm{~min}$ and Safranin-O for $5 \mathrm{~min}$. The immunochemical staining of intracellular proteins were prepared as our previously described. ${ }^{18}$ Briefly, cells were fixed with $4 \%$ paraformaldehyde and permeabilized with $0.3 \%$ Triton $\mathrm{X}-100$ for $10 \mathrm{~min}$. For aggrecan assay, cells were incubated with mouse primary antibodies against pig aggrecan (Abcam, Cambridge, MA) overnight at $4{ }^{\circ} \mathrm{C}$, followed by fluorescein-coupled antimouse IgG (American Qualex) for $1 \mathrm{~h}$ at room temperature. For collagen II assay, a collagen staining kit was used (Chondrex, Redmond, WA) according to the manufacturer's instructions. First, cells were incubated with $2 \%$ bovine testicular hyaluronidase for $30 \mathrm{~min}$, and blocking buffer for $30 \mathrm{~min}$ followed by collagen II monoclonal antibodies (Abcam, Cambridge, MA) overnight at $4{ }^{\circ} \mathrm{C}$. Then, cells were incubated in secondary antibody for $1 \mathrm{~h}$ at room temperature and developed by 3,3'-diaminobenzidine (DAB) substrate solution for $10 \mathrm{~min}$. The fluorescence images were obtained using a microscope (Leica DM6000B, Switzerland).

\section{Culture of porcine IVD cells with the NP and AF scaffolds}

Porcine IVD cells were plated onto the scaffold similarly to our published method. ${ }^{\mathbf{1 8 2 1}}$ Briefly, PBST film was cut into sections with diameter of $1.2 \mathrm{~cm}$, pre-soaked into $75 \%$ ethanol for $2 \mathrm{~h}$ followed by sterilized under ultraviolet light for $2 \mathrm{~h}$. Then, the film was washed with PBS and placed into a 24-well culture plate. The porcine IVD cells obtained as described above $(2 \times$ $10^{4}$ cells per $\mathrm{mL}$ ) was seeded in each well on PBST film for $4 \mathrm{~h}$ prior to the addition of normal culture medium, which was changed every two days. After $24 \mathrm{~h}$ of culture, live/dead cell staining was performed using the fluorescent probes calceinAM and propidium iodide (PI). The cells were washed with PBS, followed by incubation with a PBS solution mixed with 10 $\mu \mathrm{L}$ calcein-AM and $3 \mu \mathrm{L}$ PI for $15 \mathrm{~min}$. Then, cells were washed with PBS before visualization. The survival rates and proliferation activity were determined using CCK-8 cell counting kit (Sigma Aldrich, USA) according to manufacturer's instructions. After $3 \mathrm{~d}$ of culture, the cells morphologies on PBST film were stained with fluorescein isothiocyanate (FITC)-phalloidin (Sigma) for F-actin and diamidino-phenyl-indole (DAPI, Roche) for the nucleus. In addition, chitosan hydrogel scaffold was cut into sections with diameter of $1.2 \mathrm{~cm}$ and the other procedures followed as above.

\section{Preparation of tissue-engineered IVD via a combination of scaffolds and cells}

Firstly, sterile tissue-engineered IVD scaffold were immersed into DMEM for $48 \mathrm{~h}$ followed by paper drying. Then, IVD cells ( 2 $\times 10^{7}$ cell per $\mathrm{mL}$ ) were added drop-wise onto the surface of chitosan hydrogel $(200 \mu \mathrm{L})$ and PBST films $(200 \mu \mathrm{L})$. Four hours later, PBST films were rolled surrounding the central hydrogel and inserted into the PEEK scaffold. Finally, the constructs were completely immersed into the culture medium and further cultured for 2 weeks before implantation. 


\section{Surgical implantation of tissue-engineered IVD into the porcine spine}

All animal procedures were performed according to the Guidelines for Care and Use of Laboratory Animals of North Sichuan Medical College and experiments were approved by the Animal Ethics Committee of North Sichuan Medical College. Ten male Bama miniature pigs (average weight: $25 \mathrm{~kg}$ ) was used due to its large similarity with human spine in terms of geometrical and biomechanical properties. ${ }^{22}$ After anesthesia via intravenous injection of phenobarbital sodium $(10 \mathrm{mg}$ $\mathrm{kg}^{-1}$ ), the lumbar IVD were exposed via an anterior lateral approach. Then, the AF was incised, and the intact disc was removed while great care was taken to preserve the endplates (ESI Fig. S1a $\dagger$ ). The tissue-engineered IVD was inserted into the disc space, and internally fixed with titanium plates and screws (Ti-6Al-4V, Showa Ika, Co Ltd., Toyohashi, Japan) (ESI Fig. S1b广). Subsequently, the surgical incision was closed in layers and post-surgical infection treatment with penicillin was performed for 3 days.

\section{Radiographic and MRI imaging analysis of tissue-engineered IVD in vivo}

For imaging procedures, the pigs were tranquilized with an injection of $1 \%$ pentobarbital $\left(1 \mathrm{~mL} \mathrm{~kg}^{-1}\right)$. To evaluate implant stability, the lateral X-ray radiography (Multix Fusion, Siemens, Muenchen, Germany) of the lumbar spine was taken at 4 and 8 weeks after tissue-engineered IVD transplantation. Disc height index (DHI), a standard technique used to normalize disc height to vertebral body length, was quantified using a custom MATLAB program (Mathworks, Natick, USA). ${ }^{15}$ The change of DHI was expressed as followed:

$$
\% \mathrm{DHI}=\text { postoperative } \mathrm{DHI} / \text { preoperative } \mathrm{DHI} \times 100 \%
$$

Meanwhile, T2-weighted images of the lumbar spines were taken using a 3T clinical MRI scanner (Siemens Magnetom TrioTim, Munich, Germany) and quantified using Analyze 7.0 software (Analyze Direct). The \% ST2WI was defined as followed:

$\% \mathrm{ST} 2 \mathrm{WI}=$ postoperative ST2WI/preoperative ST2WI

$$
\times 100 \%
$$

\section{Sample harvesting and histological analysis}

Pigs were sacrificed at 4 and 8 weeks postoperatively, the tissueengineered IVDs were harvested, wrapped in PBS-soaked gauze, and stored in $\mathrm{a}-80{ }^{\circ} \mathrm{C}$ freezer until use. For histological analysis, samples were placed in $10 \%$ neutral buffered formalin, decalcified and processed into paraffin. Sagittal $5 \mu \mathrm{m}$-thick sections were prepared and stained using histopathology assays of standard hematoxylin-eosin (H\&E) staining, SafraninO staining as well as immunochemical staining of aggrecan and collagen II.

\section{Real-time PCR analysis of gene expression levels}

To quantify the transcription level of collagen II and aggrecan genes, tissue-engineered IVD was harvested and digested with trizol solution to collect the cells for subsequent RNA extraction. RNA extraction was performed according to the protocols specified in the RNeasy Mini Kit (Omega, USA) and reversetranscribed to cDNA using a first stand cDNA synthesis kit (Promega, Madison, WI, USA). The relative expression of collagen II and aggrecan, normalized to that of $\beta$-actin, was determined by a real-time polymerase chain reaction (PCR) based method. ${ }^{14}$

\section{Biomechanical testing}

To test the compressive stress of the tissue-engineered IVD scaffold, the samples with dimensions of $1.25 \mathrm{~cm}$ (diameter) by $2.5 \mathrm{~cm}$ (height) was measured using the testing machine at a rate of $5 \mathrm{~mm} \mathrm{~min} \mathrm{~m}^{-1}$ and stopped at $60 \%$ strain. The sample testing was repeated five times. In addition, the biomechanical properties of five tissue-engineered IVD samples obtained from the sacrificed pigs after 8 weeks were tested as the method mentioned above. Moreover, the compressive stress and elasticity modulus were compared with the normal porcine IVD.

\section{Statistical analysis}

All quantitative data are presented as means \pm standard deviation (SD). Statistical differences between two groups were calculated using a Student's $t$-test and $p<0.05$ was considered statistically significant.

\section{Results}

\section{Characterization of tissue-engineered scaffolds}

The gross appearance of the whole tissue-engineered IVD scaffold was similarly to that of the native IVD, which was composed of NP, IAF and OAF phases (Fig. 2a). The NP phase stained pink and consisted of chitosan hydrogel. SEM revealed that the inner red hydrogel possessed a highly interconnected porous structure and the pore diameter ranged from 50 to $150 \mu \mathrm{m}$ (Fig. 2b).

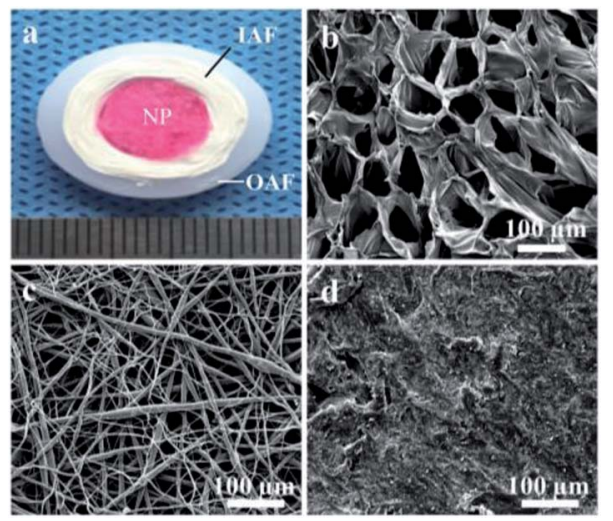

Fig. 2 (a) Gross morphology of the tissue-engineered IVD scaffold and SEM images showing the microstructure and morphology of (b) chitosan hydrogel, (c) PBST film and (d) PEEK ring. 
Surrounding the red chitosan hydrogel, the white film as the IAF phase were rolled and used to seal the NP phase. The film was composed of nanofibers which were randomly oriented and aligned (Fig. 2c). The diameters of the nanofibers had a wide range, from $200 \mathrm{~nm}$ to $2 \mu \mathrm{m}$. The porosity of the film was $61.83 \% \pm 7.33 \%$ and the water absorption was $297.34 \% \pm$ $57.13 \%$. The outermost ring with solid and rough surface was used as the OAF phase, for sustaining the hydrogel nucleus and IAF phase (Fig. 2d).

The XRD pattern of the film sample was shown in Fig. 3a. The peaks of $2 \theta$ at $17.4^{\circ}, 20.5^{\circ}$ and $23.2^{\circ}$ were characteristic peaks of polybutylene terephthalate (PBT) and polybutylene succinate (PBS). ${ }^{\mathbf{2 3} 24}$ The crystallinity of the film was approximate $45 \%$. In addition, PBST nanofibers had a high tensile strength up to $\sim 38 \mathrm{MPa}$. Also, FTIR spectrum of the film (Fig. 3b) showed that the strong peaks at 1268 and $1712 \mathrm{~cm}^{-1}$ were attributed to $\mathrm{C}=\mathrm{O}$ carbonyl stretching vibration and the peak at $728 \mathrm{~cm}^{-1}$ was attributed to $\mathrm{C}-\mathrm{H}$ bending vibration absorption of benzene, consistent with those of PBST. ${ }^{25,26}$ XPS analysis (Fig. 3c) exhibited carbon and oxygen peaks as the main atomic elements.

Moreover, the XRD pattern of the ring sample (Fig. 3d) showed that the peaks of $2 \theta$ at $18.9^{\circ}, 20.9^{\circ}$ and $22.7^{\circ}$ matched with the characteristic peaks of PEEK. ${ }^{27}$ Similarly to PBST, the semi-crystalline PEEK had a high tensile strength ( $65 \mathrm{MPa})$. Moreover, the peaks at $1652 \mathrm{~cm}^{-1}$ was attributed to $\mathrm{C}=\mathrm{O}$ carbonyl stretching vibration and the strong peaks at 1597 and $1485 \mathrm{~cm}^{-1}$ were attributed to the skeletal ring vibration of benzene (Fig. 3e). The peaks at 838 and $765 \mathrm{~cm}^{-1}$ were
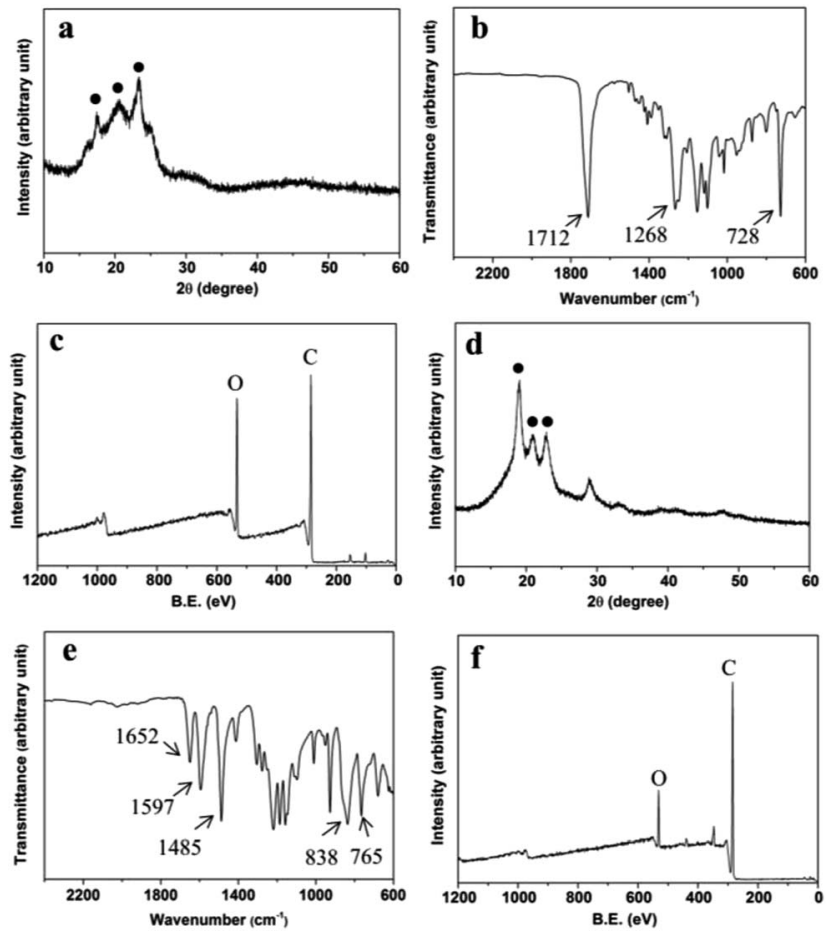

Fig. 3 (a) XRD pattern, (b) FTIR spectrum and (c) XPS spectrum of PBST film, and (d) XRD pattern, (e) FTIR spectrum and (f) XPS spectrum of PEEK ring. attributed to the $\mathrm{C}-\mathrm{H}$ bending vibration adsorption of benzene. ${ }^{28}$ Also, XPS analysis of the ring sample (Fig. 3f) showed carbon and oxygen peaks as the main atomic elements.

\section{In vitro evaluation of the isolated IVD cells}

The obtained IVD cells were observed by optical microscopy and revealed the IVD cells with a spindle-like or oval morphology (Fig. 4a). The Safranin O staining results showed that abundant proteoglycan was secreted by the IVD cells (Fig. 4b). The collagen II immunohistochemistry staining indicated that IVD cells also successfully expressed collagen II (Fig. 4c). The immunofluorescence staining images showed that aggrecan was successfully synthesized (Fig. 4d).

\section{In vitro evaluation of cells activities in the IVD scaffolds}

The calcein-AM/PI (live/dead) double staining showed that a large number of viable cells were seen in both AF film (Fig. 5a) and NP hydrogel (Fig. 5b) after culture for $24 \mathrm{~h}$. The cells were evenly distributed on the nanofibers (Fig. 5a), and around the porous structure of hydrogel (Fig. 5b). The cells survival rates were $93.8 \pm 2.2 \%$ on AF film and $95.1 \pm 2.6 \%$ on NP hydrogel, respectively (Fig. 5e). Also, fluorescent staining of IVD cells were used to observe the cell adhesion after culture for 3 days. As visualized by cytoskeleton staining using FITC-phalloidin and nuclei staining using DAPI (Fig. 5c and d), IVD cells possessed multi-polar spindle-shaped morphology with well organized cytoskeleton structures on both phase. Moreover, the IVD cells appeared to proliferate much faster on the NP phase than on the AF phase. Correspondingly, the cell growth curve on the NP and AF phase showed a similar increase tendency (Fig. 5f).

\section{In vivo evaluation of disc height and T2-weighted MRI image}

At $4^{\text {th }}$ and $8^{\text {th }}$ weeks after tissue-engineered IVD transplantation, the X-ray radiographs (Fig. 6a and b) showed good internal fixation, and no fracture and spondylolisthesis existence. Also, the vertebral space height appeared no obvious difference compared with that of the adjacent intervertebral. The adjacent IVD showed similar \% DHI of $\sim 100 \%$ throughout 8 weeks. The tissue-engineered IVD maintained a \% DHI of

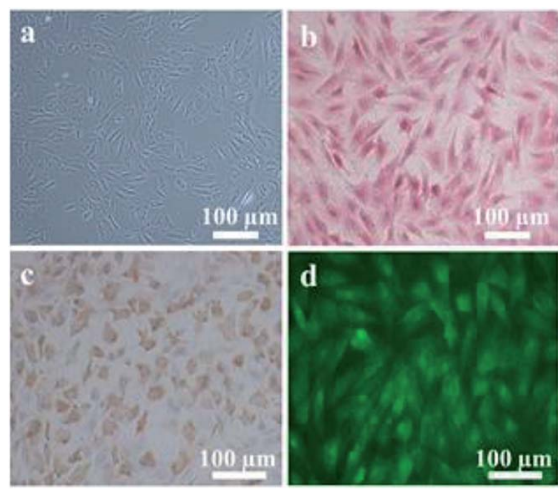

Fig. 4 (a) Light microscopy image of IVD cells morphology, (b) Safranin O staining, (c) collagen II immunohistochemistry staining and (d) aggrecan immunofluorescence staining of IVD cells. 


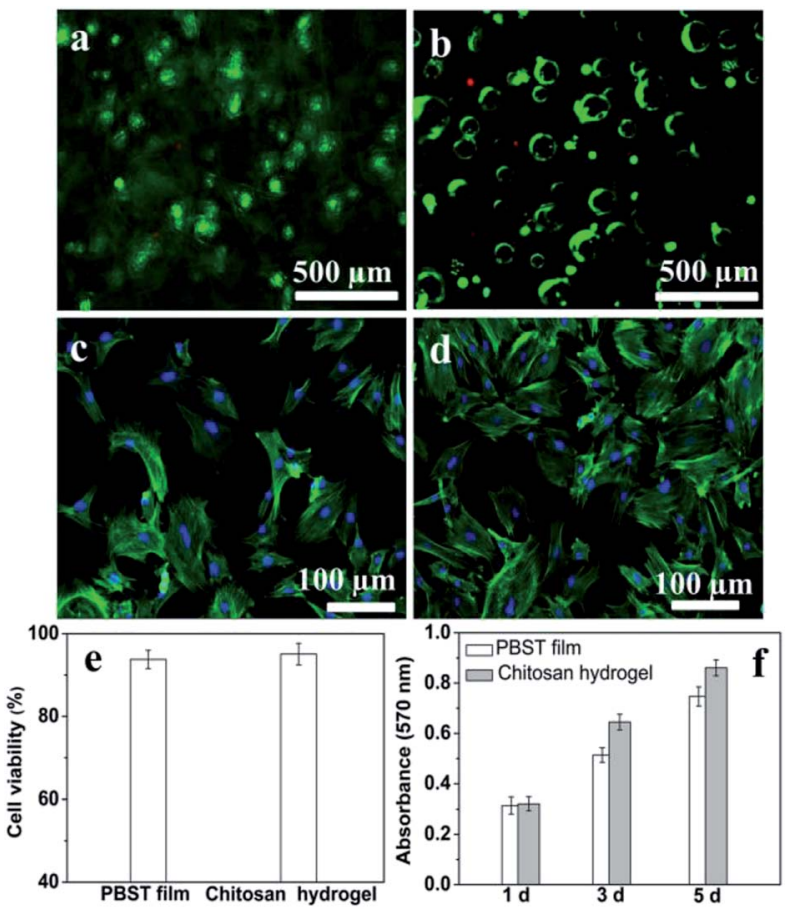

Fig. 5 Live/dead staining of IVD cells seeded on (a) the PBST film and (b) chitosan hydrogel for $24 \mathrm{~h}$ (live cells (green), dead cells (red)); fluorescence images of cell morphologies attached (c) on the PBST film and (d) chitosan hydrogel for $3 \mathrm{~d}$ (cytoskeleton filaments (green), nuclei (blue)); (e) the cell survival rate and (f) proliferation of IVD cells cultured on different scaffolds.
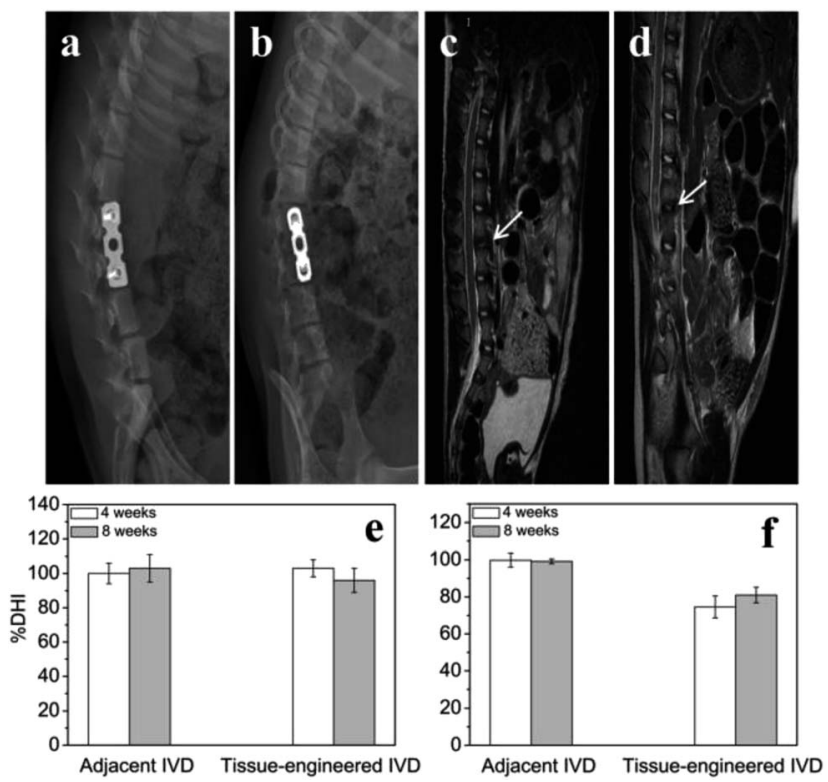

Fig. $6 \mathrm{X}$-ray radiographic images of porcine disc after tissue-engineered IVD implantation for (a) 4 weeks and (b) 8 weeks; MRI images of porcine disc after tissue-engineered IVD implantation for (c) 4 weeks and (d) 8 weeks (hydration signal (white arrow)); bar chart showing the (e) \% DHI and (f) \% ST2WI of various disc samples at different time points. $\sim 103 \%$ at 4 th week postoperation and of $\sim 96 \%$ at 8 th week postoperation. Thus, the disc height between the tissueengineered IVD and adjacent IVD had no significantly difference. Moreover, MRI results (Fig. 6c and d) showed good location of tissue-engineered IVD and normal height of the intervertebral space. The neutral region of tissue-engineered IVD showed high hydration signal intensity (white arrow) on T2-weighted images and the corresponding spinal cord had no obvious compression both at $4^{\text {th }}$ and $8^{\text {th }}$ weeks postoperation. The adjacent IVD showed similar \% ST2WI of $\sim 99 \%$ throughout 8 weeks. The tissue-engineered IVD rapidly reduced $\%$ ST2WI to $\sim 74 \%$ at 4 th week postoperation, but increased to $\sim 81 \%$ at 8 th week postoperation.

\section{Histological and immunochemical evaluation of the tissue- engineered IVD}

The tissue-engineered IVD were retrieved from the animals after culture for 8 weeks in vivo. The tissue-engineered IVD maintained their structural integrity, similarly to natural IVD in shape and tenacity (Fig. 7a). OAF scaffold was obviously visible, while the central NP was comprised of gelatinous tissue and IAF was comprised of light white fibrous tissue arranged in concentric circles. As mentioned above, OAF phase was composed of solid PEEK and had no cell growth. Thus, removing the OAF phase, only the NP and IAF phase were staining and further analyzed. H\&E and Safranin-O staining (Fig. $7 \mathrm{~b}$ and c) showed that massive amounts of cells and proteoglycan presented in the tissue-engineered IVD. Immunohistochemistry for collagen II was intensively positive for both NP and AF phases (Fig. 7d). Immunofluorescence for aggrecan (Fig. 7e) showed green fluorescence both in the NP and AF phase. Also, both porous structure of NP scaffold and layer structure of IAF phase were evidently visible (Fig. 7e).

\section{Gene expression evaluation of the tissue-engineered IVD}

The gene expression of collagen II and aggrecan in tissueengineered IVD at different time points were measured and shown in Fig. 8. The gene expression of collagen II and aggrecan in the tissue-engineered IVD were both significantly enhanced

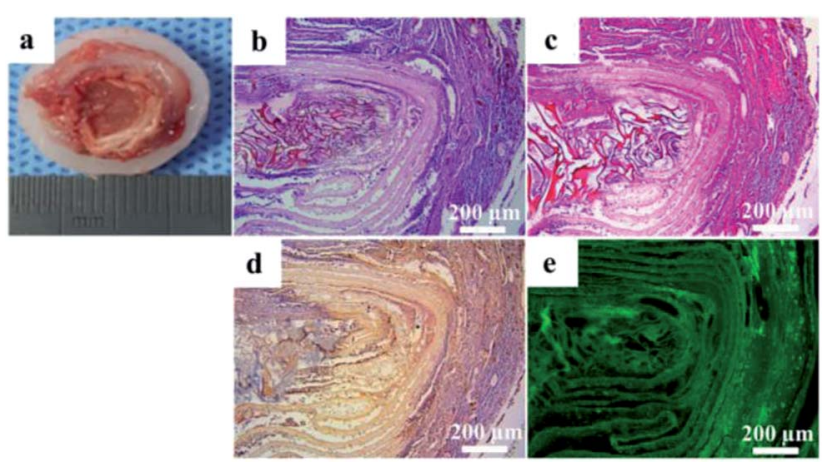

Fig. 7 (a) Gross morphology of the tissue-engineered IVD retrieved from the pig after implantation for 8 weeks; (b) H\&E, (c) Safranin-O, (d) collagen II immunohistochemistry and (e) aggrecan immunofluorescence staining of cross sections of the tissue-engineered IVD. 

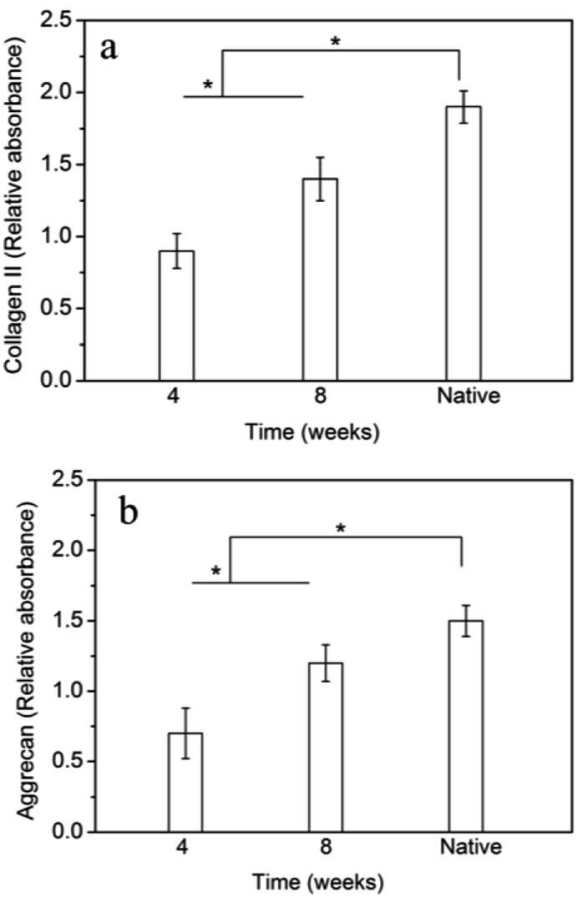

Fig. 8 RT-PCR analysis of gene expressions of (a) collagen II and (b) aggrecan of cells in tissue-engineered IVD. * indicates significant difference between the groups $(p<0.05)$.

with time increase from 4 weeks to 8 weeks. Although collagen II and aggrecan expression in the tissue-engineered IVD at 8 weeks were less than those of native IVD, the collagen II and aggrecan gene did prolong expression with time increase.

\section{The mechanical properties of tissue-engineered IVD}

The compressive stress and elastic moduli of the tissueengineered scaffold was $5.8 \pm 2.1 \mathrm{MPa}$ and $38.5 \pm 4.6 \mathrm{MPa}$, respectively (Table 1). After implantation for 8 weeks, the compressive stress and elastic moduli of the tissue-engineered IVD was $7.5 \pm 1.3 \mathrm{MPa}$ and $58.4 \pm 12.9 \mathrm{MPa}$, respectively, which was very close to those of the natural porcine IVD $(9.6 \pm$ 1.4 MPa and $71.5 \pm 18.2 \mathrm{MPa}$, respectively).

\section{Discussion}

Many previous studies have been concentrated on regenerating a specific component of IVD, including NP or AF separately. ${ }^{29-31}$ Also, the regenerative IVD cartilage endplate capable of nutrients and metabolites exchange was prepared in our previous study ${ }^{32}$ However, the separate scaffolds could not emulate the structure and mechanical properties of natural IVD. Thus,

Table 1 The compressive strength and elasticity modulus of various samples

\begin{tabular}{lll}
\hline Sample & $\begin{array}{l}\text { Compressive strength } \\
(\mathrm{MPa})\end{array}$ & $\begin{array}{l}\text { Elasticity modulus } \\
(\mathrm{MPa})\end{array}$ \\
\hline IVD scaffold & $5.8 \pm 2.1$ & $38.5 \pm 4.6$ \\
Tissue-engineered IVD & $7.5 \pm 1.3$ & $58.4 \pm 12.9$ \\
Native IVD & $9.6 \pm 1.4$ & $71.5 \pm 18.2$
\end{tabular}

a growing number of studies have focused on fabricating the composite IVD. Zhuang et al. ${ }^{33}$ construct composite IVD consisting of demineralized BMG as the NP phase and collagen II/ hyaluronate-6-sulfate as the AF phase. After implantation into mice for 12 weeks, the gross morphology and biochemical properties of the composite tissue-engineered IVD resembled those of native IVD. Also, Xu et al. ${ }^{14}$ developed an integrated biphasic IVD scaffold using BMG as OAF phase and acellular cartilage ECM as NP phase for mimicking natural IVD component and structure. In vitro and in vivo experiments demonstrated that the composite scaffold could secrete large amounts of ECM similar to native IVD. These studies suggested that fabrication of a tissue-engineered composited IVD possessing of morphological and biochemical properties similar to native IVD was feasible. However, it was noteworthy that these biomaterials applied for tissue-engineered IVD, especially for tissueengineered $\mathrm{AF}$, such as collagen, PCL, and hyaluronic acid, ${ }^{34,35}$ had poor mechanical properties for maintaining tissueengineered IVD bioactivity in large animals. Also, those tissueengineered $\mathrm{AF}$ approaches focused on constructing inner $\mathrm{AF}$ and regenerating cartilage-like tissue, while ignoring the outer AF structure. Thus, tissue-engineered IVD described in this paper, including OAF, IAF and NP structure, was constructed for IDD regeneration in large animals.

Since mechanics plays a key role in the construction and remodeling of tissue-engineered IVD in vivo, selection of appropriate biomaterials has been a major factor in constructing tissue-engineered IVD with improving mechanical properties. The whole tissue-engineered IVD described in this paper included a central chitosan hydrogel to simulate the NP structure, surrounding with a PBST fiber films. Then, an outer PEEK ring was used to simulate the OAF phase, which maintained the hydrogel nucleus and IAF structure. Firstly, as a natural cationic biopolymer, chitosan has received much attention in biomedical field due to its biocompatibility and biodegradability. Chitosan hydrogels comprised of cross-linked polymer networks have a high affinity for water, which can swell as a sponge and further maintain nutrition and cell survival. ${ }^{36}$ Thus, chitosan hydrogel could be a very appropriate candidate for NP regeneration. More importantly, the chitosan hydrogel in this study had excellent physicochemical and biochemical properties due to the usage of a novel crosslinker according to our previous report. ${ }^{18}$ Secondly, as aliphatic-aromatic polyesters, PBST has received much attention due to its desirable mechanical properties and biodegradability. ${ }^{37,38}$ Also, the biodegradability and physical properties of PBST can be adjusted by varying the molar ratio of comonomers. In this study, PBST was made into nanofibers, mimicking the collagenous fiber structure of natural IVD. Moreover, the high porosity $(61.83 \% \pm 7.33 \%)$ and water absorption $(297.34 \% \pm 57.13 \%)$ of the nanofibers are beneficial to the nutrients transport and metabolites excretion. ${ }^{39}$ Comparing with the biomaterials commonly used for AF regeneration, such as silk, collagen, polylactic acid (PLA) and polyurethane, the PBST in this study demonstrated super mechanical properties. The tensile strength of PBST was $\sim 38 \mathrm{MPa}$ and the elastic modulus was $\sim 650 \mathrm{MPa}$, which were much higher than those of PLA and poly(lactic-co-glycolic acid) 
(PLGA) (tensile strength: 21-29 MPa, elastic modulus: 447-453 $\mathrm{MPa}) .{ }^{40}$ In addition, we demonstrated that the IVD cells cultured on the chitosan hydrogel and PBST fibers showed a good cell viability and proliferation ability. Thirdly, solid PEEK ring was used as the OAF phase, for sustaining the hydrogel nucleus and IAF phase (Fig. 2d). As a semi-crystalline and non-resorbable thermoplastic polymer, PEEK has been widely used in clinical applications such as total join replacement, spinal cage fusion and craniomaxillofacial reconstruction, due to its excellent biocompatibility and mechanical properties. ${ }^{41,42}$ Compared with titanium and other metallic alloys, PEEK has lower elastic modulus similar to cortical bone, which reduces the extent of stress shielding after implantation. In this study, the tensile strength of PEEK was $\sim 65 \mathrm{MPa}$ and the elastic modulus was $\sim 3460 \mathrm{MPa}$. Indeed, the incorporation of the PEEK ring significantly increased the compressive strength of the chitosan hydrogel/PBST fiber composite, which was observed during the mechanical testing process. Therefore, the chitosan hydrogel nucleus, PBST fiber and PEEK ring were assembled into scaffolds and further combined with cells for constructing tissueengineered IVD. As mentioned before, the tissue-engineered IVD had similar morphological structure to natural IVD.

In this study, the whole tissue-engineered IVD was further transplanted into the porcine lumbar spine. In previous studies, tissue-engineered IVDs had always been performed in vivo in the small animal model, such as Sprague Dawley rats and rabbits. ${ }^{\mathbf{4}, 15}$ Although tissue-engineered IVD showed promising performance at maintaining disc height and large amounts of ECM secretion, the primary limitation of these models was that the physiologic loading environment had a significant difference with human IVD. Also, there was anatomical difference between rat tail vertebrae and human IVD, as rat tail vertebrae lack spinal canal, posterior bone as well as joint elements. Thus, to move tissue-engineered IVD toward clinical application, pig, which has similar anatomical features and biomechanical properties with human IVD, was utilized in this study. ${ }^{22,43}$ After tissue-engineered IVD transplantation into porcine for 8 weeks, it was concluded that the dis height between tissue-engineered IVD and adjacent IVD had no significantly difference, suggesting that tissue-engineered IVD implantation could preserve the disc height in the case of discectomy as the normal disc height. Also, the disc height maintenance was also attributed to external fixation according to the results reported by Martin et $a .^{15}$ Moreover, MRI results indicated that the tissueengineered IVD implantation could maintain large extracellular matrix and water content in NP compared with the normal disc, and restrain disc degeneration effectively. From the histological and immunochemical staining, it was concluded that cells distributed and grew well inside the tissue-engineered scaffold, and further sustainably secreted ECM (including collagen II and aggrecan). All these indicated that the tissueengineered IVD had similar morphological and histological structure to natural IVD. Moreover, after implantation for 8 weeks, the compressive stress and elastic moduli of the tissueengineered IVD was strengthened in the porcine physiological environment. In contrast, the corresponding mechanical properties of tissue-engineered composite IVD were found to decrease with culture time increasing in nude mice, according to the study reported by Zhuang et al. ${ }^{44}$ We speculated that on the one hand, the mechanical stimulus from porcine loading environment played an important role in the remodeling process of tissue-engineered IVD, even though the external fixation might unload some portion of mechanical stress when preventing displacement of the implant. On the other hand, the secretion of ECM and new tissue formation further enhanced the mechanical properties of tissue-engineered IVD. Therefore, the tissue-engineered IVD prepared in this study could effectively achieve the IDD regeneration in the porcine lumbar spine.

Although the mechanical properties of the tissue-engineered IVD were lower than those of native IVD, it did indeed demonstrate a good performance in porcine IVD repair. Notably, there are still many challenges need to be overcome. Firstly, the mechanical properties of scaffold biomaterials mentioned above could be improved by variation of the molar ratio of comonomers, structure modification or surface functionalization. Also, the mechanical properties of tissueengineered IVD, such as bending, tension and torsion, are needed to be further investigated. Secondly, the tissueengineered IVD herein mainly focused on the NP and AF phase, however, cartilage endplate, as the interface between the disc and vertebral body, plays important roles in nutrient supply and waste removal. Thus, the whole tissue-engineered IVD, including NP, AF and cartilage endplate, would be fabricated and integrated in the following study. Last but not least, it would be desirable to add growth factors into the scaffold for promoting the ECM secretion and new tissue formation, which may further enhance the biofunction of tissue-engineered IVD in vivo.

\section{Conclusion}

In this study, a novel whole tissue-engineered IVD was successfully fabricated for IDD regeneration in the porcine lumbar spine. The tissue-engineered scaffold was composed of an outer ring of PEEK, an inner layer of PBST fibers and a central phase of chitosan hydrogel. In vitro cell experiments demonstrated that the novel scaffolds provided an appropriate environment for supporting IVD cells growth. Furthermore, the tissue-engineered IVD constructed by seeding porcine IVD cells into the scaffolds was used for porcine IDD regeneration. In vivo testing showed that the gross morphology and biological functions of the tissue-engineered IVD were similar to those of natural porcine IVD. Thus, the novel tissue-engineered IVD could be a potential candidate for human IDD regeneration.

\section{Conflicts of interest}

There are no conflicts to declare.

\section{Acknowledgements}

This study was supported by National Natural Science Foundation of China (81171472), Applied Fundamental Program of Science \& Technology Department of Sichuan Province 
(2016JY0123, 2018JY0100), Technology Innovation Seeding Project of Sichuan Province (2017070), Applied Program of Health and Family Planning Commission of Sichuan Province (17PJ194, 17PJ208, 18PJ476), Key Project of Education Department of Sichuan Province (17ZA0178) and Science \& Technology Project of Nanchong City (NSMC20170310, NSMC20170453 and NSMC20170203).

\section{References}

1 M. Gore, A. Sadosky, B. R. Stacey, K.-S. Tai and D. Leslie, Spine, 2012, 37, E668-E677.

2 S. Dagenais, J. Caro and S. Haldeman, Spine J., 2008, 8, 8-20.

3 H. J. Han, H. Y. Yoon, J. Y. Kim, H. Y. Jang, B. Lee, S. H. Choi and S. W. Jeong, Am. J. Chin. Med., 2010, 38, 1015-1025.

4 U. S. Van, J. Silva-Correia, V. M. Correlo, J. M. Oliveira and R. L. Reis, Biofabrication, 2015, 7, 015008.

5 Z. Sun, B. Luo, Z. H. Liu, D. Samartzis, Z. Liu, B. Gao, L. Huang and Z. J. Luo, Int. J. Biol. Sci., 2015, 11, 133-143.

6 B. Yue, Y. Lin, X. Ma, G. Zhang and B. Chen, Mol. Med. Rep., 2016, 14, 4593-4598.

7 H. Tao, Y. Wu, H. Li, C. Wang, Y. Zhang, C. Li, T. Wen, X. Wang, Q. He and D. Wang, ACS Appl. Mater. Interfaces, 2015, 7, 17076-17087.

8 C. Fernandez, A. Marionneaux, S. Gill and J. Mercuri, J. Biomed. Mater. Res., Part A, 2016, 104, 3093-3106.

9 T. Wen, C. Zhang, D. Ruan, F. Li and J. Ma, J. Biomater. Tiss. Eng., 2014, 4, 1073-1079.

10 D. H. Kim, J. T. Martin, D. M. Elliott, L. J. Smith and R. L. Mauck, Acta Biomater., 2015, 12, 21-29.

11 H. Cho, S. H. Park, K. Park, J. W. Shim, J. Huang, R. Smith, S. Elder, B. H. Min and K. A. Hasty, Artif. Organs, 2013, 37, E131-E138.

12 N. Wismer, S. Grad, G. Fortunato, S. J. Ferguson, M. Alini and D. Eglin, Tissue Eng., Part A, 2014, 20, 672-682.

13 J. Silva-Correia, S. I. Correia, J. M. Oliveira and R. L. Reis, Biotechnol. Adv., 2013, 31, 1514-1531.

14 B. Xu, H. Xu, Y. Wu, X. Li, Y. Zhang, X. Ma and Q. Yang, PLoS One, 2014, 10, e0124774.

15 J. T. Martin, A. H. Milby, J. A. Chiaro, D. H. Kim, N. M. Hebela, L. J. Smith, D. M. Elliott and R. L. Mauck, Acta Biomater., 2014, 10, 2473-2481.

16 Y. Moriguchi, J. Mojica-Santiago, P. Grunert, B. Pennicooke, C. Berlin, T. Khair, R. Navarro-Ramirez, R. J. Arbona, J. Nguyen, R. Hartl and L. J. Bonassar, PLoS One, 2017, 12, e0185716.

17 G. D. O'connell, E. J. Vresilovic and D. M. Elliott, Spine, 2007, 32, 328-333.

18 Z. Chen, M. Zhao, K. Liu, Y. Wan, X. Li and G. Feng, J. Mater. Sci.: Mater. Med., 2014, 25, 1903-1913.

19 S. Shao, S. Zhou, L. Li, J. Li, C. Luo, J. Wang, X. Li and J. Weng, Biomaterials, 2011, 32, 2821-2833.
20 J. Melrose, S. Smith and P. Ghosh, Eur. Spine J., 2003, 12, 5765.

21 Y. Wan, G. Feng, F. H. Shen, C. T. Laurencin and X. Li, Biomaterials, 2008, 29, 643-652.

22 I. Busscher, J. J. Ploegmakers, G. J. Verkerke and A. G. Veldhuizen, Eur. Spine J., 2010, 19, 1104-1114.

23 K. J. Ihn, E. S. Yoo and S. S. Im, Macromolecules, 1995, 28, 2460-2464.

24 Y. Ichikawa, H. Kondo, Y. Igarashi, K. Noguchi, K. Okuyama and J. Washiyama, Polymer, 2000, 41, 4719-4727.

25 R. Hajiraissi and M. Parvinzadeh, Appl. Surf. Sci., 2011, 257, 8443-8450.

26 T. Okajima, K. Hara, M. Yamamoto and K. Seki, Polymer, 2012, 53, 2956-2963.

27 L. Wang, S. He, X. Wu, S. Liang, Z. Mu, J. Wei, F. Deng, Y. Deng and S. Wei, Biomaterials, 2014, 35, 6758-6775.

28 A. G. A. Lafi, Polym. Degrad. Stab., 2014, 105, 122-133.

29 S. Wan, S. Borland, S. M. Richardson, C. L. Merry, A. Saiani and J. E. Gough, Acta Biomater., 2016, 46, 29-40.

30 E. A. Growney Kalaf, R. Flores, J. G. Bledsoe and S. A. Sell, Mater. Sci. Eng., C, 2016, 63, 198-210.

31 B. K. Bhunia, D. L. Kaplan and B. B. Mandal, Proc. Natl. Acad. Sci. U. S. A., 2018, 115, 477-482.

32 D. Yuan, Z. Chen, Y. Zhou, D. Xiao, K. Liu, X. Xiang, L. Deng, H. Dong and G. Feng, Spine, 2017, 42, E260-E266.

33 Y. Zhuang, B. Huang, C. Q. Li, L. T. Liu, Y. Pan, W. J. Zheng, G. Luo and Y. Zhou, Biochem. Biophys. Res. Commun., 2011, 407, 327-332.

34 N. L. Nerurkar, S. Sen, A. H. Huang, D. M. Elliott and R. L. Mauck, Spine, 2010, 35, 867-873.

35 A. T. Choy and B. P. Chan, PLoS One, 2015, 10, e0131827.

36 N. C. Cheng, W. J. Lin, T. Y. Ling and T. H. Young, Acta Biomater., 2017, 51, 258-267.

37 S. Luo, F. Li, J. Yu and A. Cao, J. Appl. Polym. Sci., 2012, 125, 2426-2432.

38 P. H. Tsai, C. H. Wang, L. S. Kan and C. W. Chen, Asia-Pac. J. Chem. Eng., 2012, 7, S88-S94.

39 A. Abdal-Hay, K. H. Hussein, L. Casettari, K. A. Khalil and A. S. Hamdy, Mater. Sci. Eng., C, 2016, 60, 143-150.

40 C. M. Agrawal and R. B. Ray, J. Biomed. Mater. Res., 2001, 55, 141-150.

41 P. R. Monich, B. Henriques, A. P. N. de Oliveira, J. C. M. Souza and M. C. Fredel, Mater. Lett., 2016, 185, 593-597.

42 I. V. Panayotov, V. Orti, F. Cuisinier and J. Yachouh, J. Mater. Sci.: Mater. Med., 2016, 27, 118-129.

43 I. Busscher, V. D. V. Aj, J. H. van Dieën, I. Kingma, G. J. Verkerke and A. G. Veldhuizen, Spine, 2010, 35, 35-42.

44 Y. Zhuang, J. M. Chen, C. C. Zhang, Z. Q. Li, M. Yang, C. Q. Li, B. Huang and Y. Zhou, Chin. J. Traumatol., 2015, 31, 738-742. 